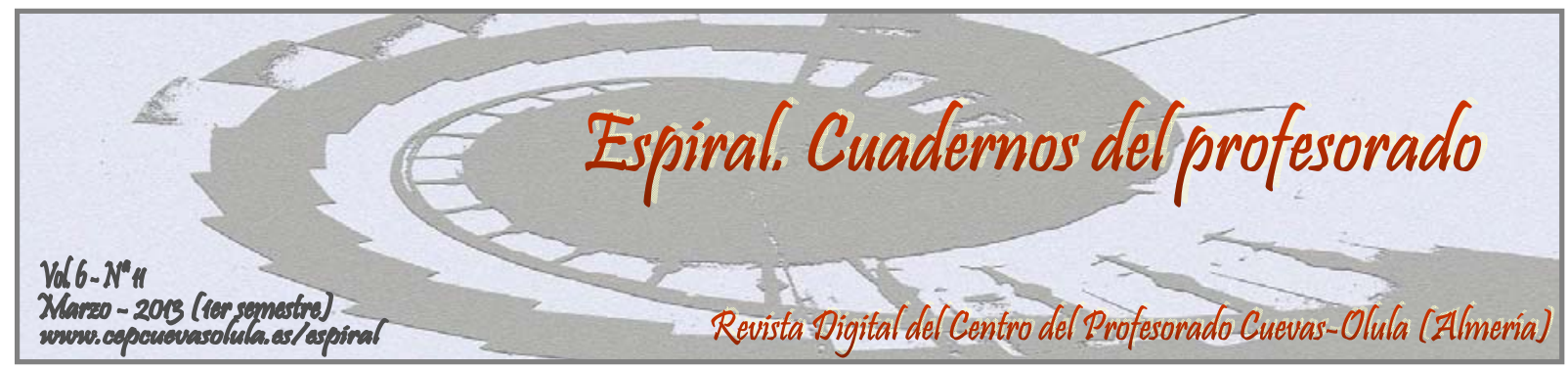

\title{
act.musica: UNA HERRAMIENTA INFORMÁTICA AL SERVICIO DEL PROFESOR DE MÚSICA
}

act.musica: A SOFTWARE TOOL IN THE SERVICE OF MUSIC TEACHERS

\section{Emilia Martos Sánchez}

\author{
Profesora de Música en el I.E.S. Rosa Navarro (Olula del Río), Almería, España
}

RESUMEN: Entre los distintos desafíos que presenta hoy día la educación, se erige como una cuestión fundamental el saber incluir las nuevas tecnologías en la práctica diaria del aula, así como convertirla en un objeto indispensable para el profesorado. El objetivo fundamental del trabajo es determinar cuáles son los aspectos más significativos del currículum así como de la programación, a los que debe dar respuesta el profesor de música de secundaria. Para ello, hemos desarrollado una herramienta informática, act.musica. El estudio combina tanto la metodología cualitativa como la cuantitativa, utilizando como técnicas de investigación, los grupos de discusión, el análisis de contenido y el cuestionario. Así pues, el análisis que realiza act.musica, nos permite esquematizar y visualizar perfectamente la representatividad de los elementos constituyentes del currículum musical de la LOE, y de las distintas programaciones, así como en un futuro establecer una posible validación cruzada.

Palabras clave: currículum, LOE, nuevas tecnologías.

ABSTRACT: Among the different challenges of education nowadays, to know how to include new technologies in daily classroom practice stands as a major issue and in fact, turning it into an indispensable object for teachers. The main objective of this study is to determine which are the most significant aspects of the curriculum and programming the high school music teacher should answer. For this, we have designed a software tool, act.musica. The study combines both qualitative and quantitative methodology, using as research techniques, focus groups, content analysis and questionnaire. The act.musica analysis allows us to perfectly sketch and visualize the most outstanding the constituent elements of the music curriculum of LOE and of the different programmings, as well as, to establish in a future a possible cross-validation.

Key words: Curriculum, LOE, new technologies.

Martos Sánchez, M. (2013). act.musica: una herramienta informática al servicio del profesor de música. Espiral. Cuadernos del Profesorado, 6(11), 12-18. Disponible en: http://www.cepcuevasolula.es/espiral

Fecha de recepción: 03/05/2012

Fecha de aceptación: 22/10/2012
Enviar correspondencia a: martosemi@gmail.com 


\section{1.- INTRODUCCIÓN}

Las tecnologías de la información y la comunicación se han convertido en un elemento clave en la sociedad de hoy día. En efecto, han llegado a producir importantes cambios en la forma de organización social y económica.

Por otro lado, existe un compromiso decidido por parte de la actual ley educativa (LOE, 2006) con los objetivos planteados por la Unión Europea y la UNESCO donde se pretende facilitar el acceso generalizado a los sistemas de educación y formación y abrir éstos al mundo exterior. Esto conlleva garantizar el acceso de todos/as a las tecnologías de la información y comunicación, orientando el sistema hacia la sociedad de conocimiento mediante la puesta en marcha de diferentes planes.

Así pues, las distintas administraciones, conscientes de lo que supone este reto han puesto en funcionamiento la Web 2.0. En Andalucía, también se ha asumido la necesidad inaplazable de reorientar nuestro sistema educativo y contribuir a la modernización del mismo y se ha hecho de manos del Plan Escuela TIC 2.0.

Sin embargo, tanto en el área de música como en el resto de materias es una realidad constatable que con la presencia de la informática en las aulas, y ahora en los hogares, no será suficiente para mejorar la calidad educativa salvo que apostemos por su integración en los procesos de enseñanza-aprendizaje y en la organización del centro (Aguaded y Tirado, 2008).

Así pues, la investigación en la que estamos trabajando y que a continuación mostramos se encuadra dentro de este contexto. En efecto, a través de ésta pretendemos ofrecer al profesorado de música, herramientas tecnológicas de útil aplicación en el ejercicio de su actividad docente. act.musica da respuesta a nuestra investigación y se adecua a dicho propósito.

Para ello, hemos dividido el artículo en diferentes apartados. En primer lugar, abordamos el planteamiento de la investigación, donde se detallan los objetivos de la misma así como las técnicas utilizadas. En segundo lugar, se describe la herramienta informática diseñada en esta investigación, tanto en su primera fase, como en la fase en la que actualmente se está trabajando. Finalmente, se establecen una serie de conclusiones al respecto, y se adjunta la relación de bibliografía detallada.

\section{Planteamiento de la Investigación}

El estudio en el que actualmente estoy trabajando surge dentro del marco de la investigación que estoy realizando para mi tesis doctoral ${ }^{1}$.

\section{Objetivos}

El objetivo fundamental del trabajo es determinar cuáles son los aspectos más significativos del currículum así como de la programación, a los que debe dar respuesta el profesor de música de secundaria, y en un futuro, cruzar ambos resultados.

Para llegar a tal fin, nos hemos propuesto una serie de objetivos a cumplir:

1. Analizar el modelo de educación musical de la LOE.

2. Conocer la formación inicial del profesorado.

3. Conocer la formación en nuevas tecnologías del profesorado.

4. Delimitar cuáles son los aspectos más significativos del currículum musical en la ESO.

5. Determinar los elementos claves de la programación didáctica utilizada por el profesor de música de secundaria.

\section{Planteamiento del problema}

El problema inicial que nos planteamos era si la formación inicial condicionaba o delimitaba el desarrollo de los contenidos, más en un área donde los profesores que imparten clase responden a una clara dualidad en lo que a su formación respecta. Así pues, en la mayor parte de los casos, el profesor

\footnotetext{
${ }^{1}$ Dirigida por Dña. Julia Bernal Vázquez, catedrática de Escuela Universitaria (Granada).
} 
de música de secundaria es o bien licenciado en Historia y Ciencias de la Música, o bien Titulado Superior de Música en sus diversas especialidades.

La posibilidad de que atendiendo a su formación inicial se incidiesen en unos contenidos curriculares u otros, nos llevó a plantearnos utilizar una de las estrategias metodológicas más habituales para la obtención de información de la realidad que se estudia: los grupos de discusión (Rincón y otros, 1995). Éstos han estado formados por un grupo de profesores de música de secundaria de la provincia de Almería.

Así pues, a través de conversaciones de grupo se han puesto en contacto y se han confrontado diferentes puntos de vista, a través de un proceso abierto en torno al tema propuesto. En concreto, la finalidad de los mismos ha sido conocer cómo se están llevando a cabo los contenidos del currículum de música, para así poder unificar criterios a la hora de elaborar la programación docente, y además proponer la necesidad de conocer cuáles son los aspectos más significativos (y en qué medida) en el currículum de música de educación secundaria obligatoria, según el sistema educativo que plantea la LOE.

Ante la disparidad de criterios y prácticas que se han podido observar en estos grupos, optamos por continuar con la investigación decantándonos por una metodología cualitativa, ya que consideramos que nuestro planteamiento encaja perfectamente dentro de las características necesarias para precisar un enfoque metodológico de este tipo (Ruiz, 2003). Por lo tanto, y dado el poco tiempo que se le dedica a la Música, y lo extenso de la propuesta curricular existente, se decidió comenzar por un análisis de contenido, técnica que nos permitiera "formular, a partir de ciertos datos, inferencias reproducibles y válidas que puedan aplicarse a su contexto” (Krippendorff, 1990). En concreto, realizamos un análisis de contenido temático, tomando como documento base el Real Decreto 1631/2006 por el que se establecen las enseñanzas mínimas de la educación secundaria obligatoria. No obstante, nuestra idea inicial fue llevar a cabo el análisis partiendo de la Orden de 10 de agosto de 2007 por la que se establecen dichas enseñanzas en Andalucía, sin embargo esto no fue posible, ya que la comunidad andaluza no ha desarrollado el currículum de música para secundaria. Para ello, se desarrolló una herramienta informática, a la que hemos denominado act.musica.

El dinamismo que ofrece la herramienta nos ha permitido poder estar trabajando en una segunda fase de la misma, la cual intenta dar respuesta a:

- a necesidad de determinar los aspectos más relevantes de las programaciones didácticas, y

- la posibilidad de ofrecer al profesorado aplicaciones tecnológicas para su uso en el aula.

En efecto, en esta segunda etapa de la investigación la metodología utilizada será doble, es decir, haremos uso de un enfoque cualitativo versus cuantitativo. Así pues, muchos autores, entienden que ambas metodologías son compatibles, e incluso recomiendan una combinación en aquellos casos que la reclamen, lo cual es conocido como triangulación (Ruiz, 2003).

La primera parte de esta segunda fase, aún en proceso, utilizará nuevamente como técnica, el análisis de contenido, facilitado por la misma herramienta (act.musica).

Paralelamente a ello, estamos elaborando un cuestionario. Este instrumento, que es el más usado en cuanto a recogida de datos, se conforma por una serie de preguntas que el encuestado deberá contestar. Estas preguntas responden a bloques claramente delimitados. Aunque aún no está totalmente finalizado y validado, podemos adelantar que sus principales secciones serán los siguientes:

- Datos personales y académicos

- Formación inicial del profesorado

- Formación permanente del profesorado

- Elaboración y uso de la programación

- Utilización de las nuevas tecnologías

- Actitud hacia las nuevas tecnologías

- Etc. 
Tal como señala Ghigliona y Mataron (1978, citado por Rodríguez, 2006), entre los tres objetivos fundamentales de éste se encuentra el que nosotros pretendemos alcanzar: contrastar nuestras hipótesis, bajo la forma de relaciones entre dos o más variables.

\section{2. act.musica: LA INFORMÁTICA AL SERVICIO DEL PROFESOR DE MÚSICA}

Fase I. El análisis del currículum musical de la ESO en la LOE

\section{Descripción de las características de la herramienta informática diseñada}

act.musica es una herramienta parametrizable y dinámica que permite interactuar con el sistema, para ajustar el estudio cualitativo a nuestras necesidades o inquietudes (Figura 1).

La aplicación esta dividida en dos partes diferenciadas: el análisis de contenido y la categorización temática.

a) El análisis de contenido:

El análisis de contenido, permite configurar varios parámetros antes de ejecutar el estudio, de modo que el ajuste de los mismos posibilite el afinamiento de los resultados obtenidos. Así pues, podemos distinguir tres tipos de ajustes, el de las stops words, el del umbral de importancia de los términos, así como el número de contextos asociados a

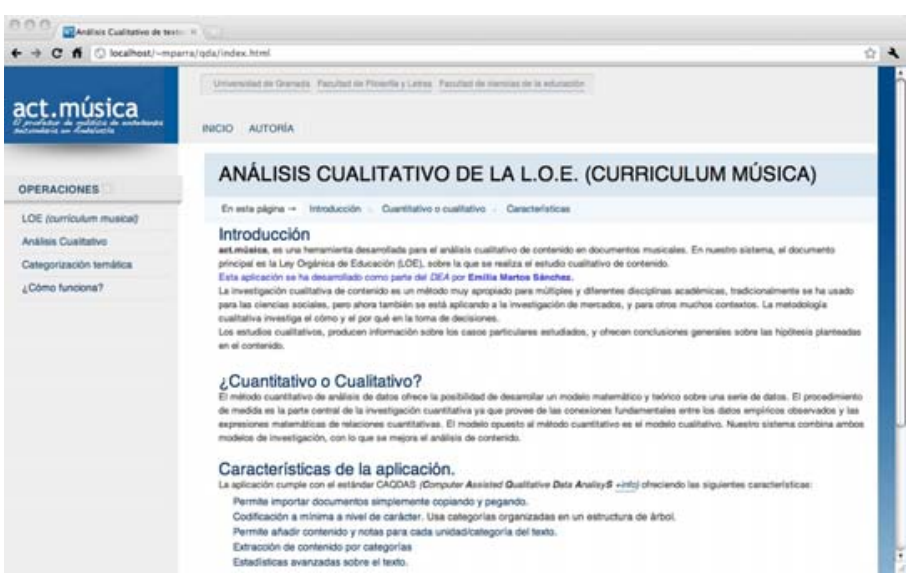

Figura 1. Aspecto inicial de la aplicación cada término destacado por la aplicación (Figura 2):

- Ajuste de las StopWords: Se refiere a las palabras o términos del lenguaje, que no se analizarán en un texto, puesto que no ofrecen un significado a un contexto o bien no tienen relevancia en el documento analizado. Por ejemplo: a, los, de, entre, etc.

- Umbral de importancia de los términos: El umbral de importancia, establece el límite inferior a partir del cual la importancia del término se analizará. Si el umbral es muy alto, se perderá detalle y si es muy bajo, el estudio se complicará por el número elevado de términos a analizar. Nosotros hemos establecido por defecto el umbral diez.

- Número de contextos: Establecen el máximo número de contextos que se visualizarán para cada término encontrado. Así pues, este elemento nos permitirá comprender el significado real del término resaltado, y ver a qué se refiere realmente, indicando el contexto exacto del mismo. Por defecto, hemos establecido siete.

Una vez configurado el sistema de acuerdo a nuestras exigencias, podemos proceder a la ejecución del análisis. Se comienza a procesar el texto y el tiempo que tardará en ejecutarlo, dependerá de los parámetros que anteriormente hemos comentado. Una vez terminado el análisis aparecen los resultados de una forma clara e intuitiva (Figura 3).

A la izquierda, como se

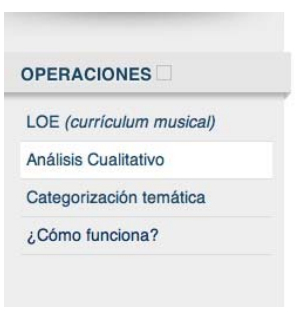

ANÁLISIS CUALITATIVO DE LA L.O.E.

En esta página $\rightarrow$ Configuración $\quad$ Ejecución del análisi

Configuración para el análisis cualitativo. Stop Words (?) (palabras o grupos de palabras no analizadas) (Ver/Modificar) Umbral de importancia〈? del los términos (Seleccionado 10) (Ver/Modificar) Número de contextos a relacionar 〈?\} (Seleccionado 7) (Ver/Modificar)

Figura 2. Elementos para configurar el análisis de contenido encontrados en el texto. A la derecha, para cada uno de los términos que aparecen se pueden consultar, por un lado, los contextos más importantes donde aparece el término o la expresión, cuyo número dependerá de la configuración inicial (Analizar contextos más relevantes) (Figura 4). 
Por otro lado, es posible conocer las relaciones de cada término con otros (Figura 5) y de este modo establecer relaciones de posible dependencia (Árbol de términos relacionados).

Así pues, los informes finales que resultan de la ejecución permiten extraer conclusiones sobre el texto estudiado, por lo que la herramienta es un medio a través del cual se pueden generar nuevo conocimiento, tal como pretendíamos.

\section{b) Categorización temática}

La segunda parte importante

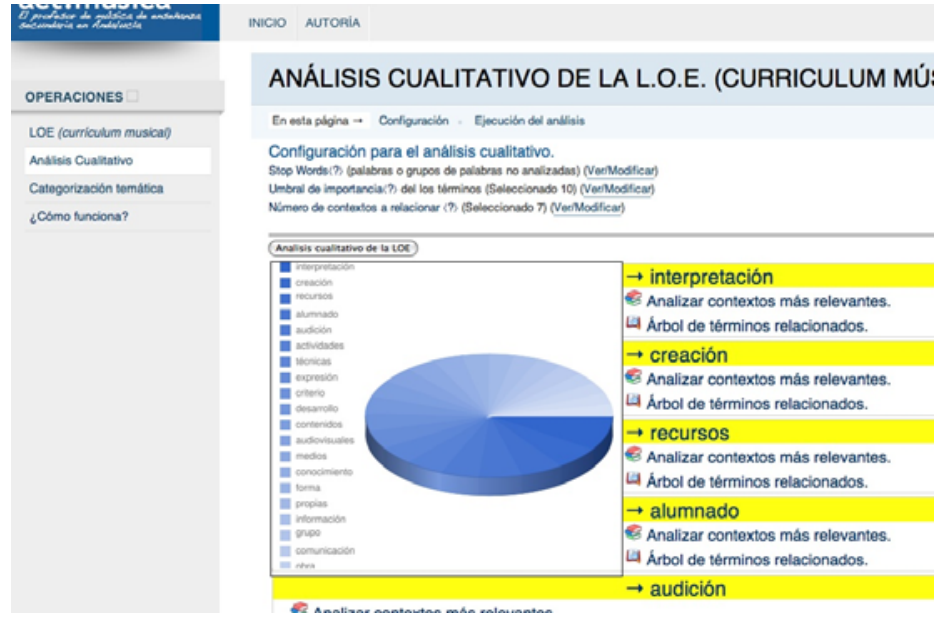

Figura 3. Apariencia de la ejecución del sistema del sistema es la que hace referencia a la categorización temática. En la sección de categorización temática, se puede consultar cada uno de los diferentes niveles de categorías que contiene el documento estudiado (Figura 6).

Como se podrá comprobar, se puede dar el caso, de que alguno de los términos destacados no encuentre términos relacionados con el mismo. Esto ocurre porque el sistema no encuentra nexos con otros términos en su base de conocimiento. No obstante, se puede subsanar, mejorando su aprendizaje mediante la incorporación de nuevos nodos, lo cual hace aún más interesante si cabe el sistema, ya que si éste estuviese disponible para todo el profesorado, su nivel de conocimiento podría implementarse de forma considerable gracias a las aportaciones de los expertos, es decir, en este caso, los profesores de música.

\section{Definición de las categorías temáticas seleccionadas}

En nuestro caso, y dado que el área de Música se estructura en dos ejes desde la educación primaria, fue esa nuestra guía para definir las categorías temáticas. Así pues, seguimos las pautas que la misma ley indica para llevar a cabo nuestro análisis de contenido, con lo cual nuestras unidades temáticas estarían determinadas por lo revisado en nuestro marco teórico.

Partiendo de esto, hemos introducido en la aplicación informática un vocabulario musical estructurado según las categorías anteriormente citadas que favorecen la base de conocimiento de la aplicación, la cual puede ser enriquecida a posteriori para una mejora del análisis de contenido (Martín, 2002).

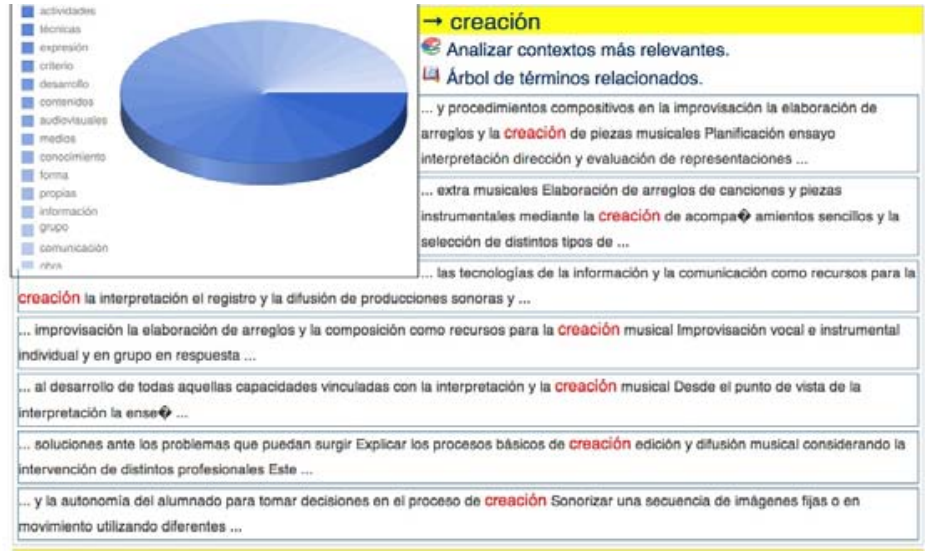

Figura 4. Ejemplo de un listado de contextos asociados a un término relevante

\section{Fase II. Análisis y evaluación de la programación didáctica}

Nuestro siguiente paso, aun en fase de pruebas, tiene como objetivo descubrir de una forma rápida y accesible a todos/as, cuáles son los elementos claves y representativos de la programación utilizada por el profesor de música. 
En esta fase, por un lado, y como anticipaba en el planteamiento de la investigación, la herramienta vuelve a hacer un análisis de contenido, utilizando los mismos ajustes que en la primera etapa (stop words, umbral de importancia, número de contextos), así como la misma categorización temática.

Por otro lado, act.musica se abre en esta nueva etapa al profesorado, permitiéndole que suba el documento que contiene la programación (en varios formatos como son .pdf, .doc o .odt) y al mismo tiempo

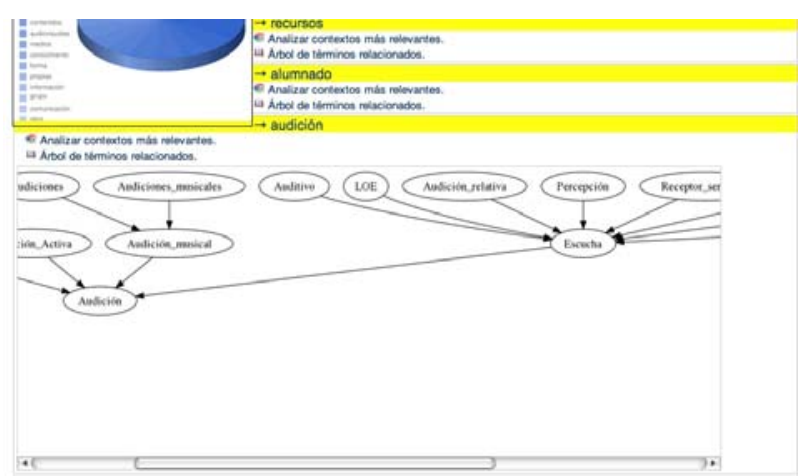

Figura 5. Relaciones que el sistema establece en base a su conocimiento previo le invita a responder a un cuestionario. Éste nos permitirá establecer una serie de conclusiones una vez que el sistema se ponga en funcionamiento, pero que fundamentalmente nos permitirá la comparación directa entre los individuos que formaran parte de nuestra muestra (Hopkins, 1989).

Finalmente, hemos de señalar que la aplicación, disponible en http://dicits.ugr.es/act.musica/, se ha desarrollado utilizando una plataforma $\mathrm{Web}^{2}$, lo que permite que sea utilizada por multitud de personas. Para todo su desarrollo e implementación sólo se han usado elementos de software libre.
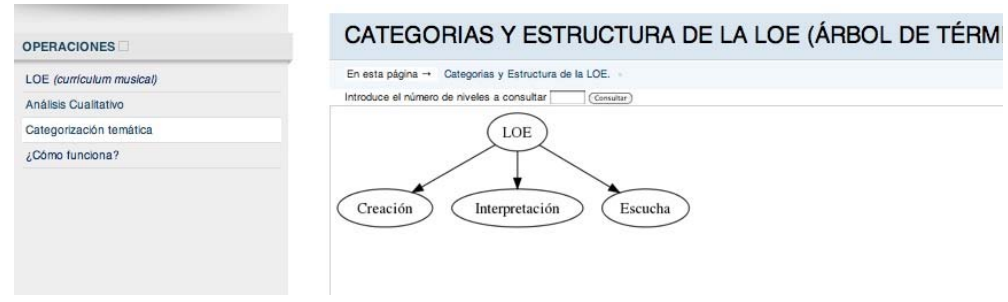

Figura 6. Ejemplo de uno de los niveles de la categorización temática

\section{CONCLUSIONES}

El análisis realizado por la herramienta que hemos creado nos permite apreciar de un solo vistazo cuáles son los términos que obtienen un mayor protagonismo en las enseñanzas mínimas establecidas en la LOE en el área de música de la etapa de secundaria.

Por orden, encontraríamos entre los quince primeros: la interpretación, la creación, los recursos, el alumnado, la audición, las actividades, las técnicas, la expresión, el criterio, el desarrollo, los contenidos, y finalmente, el término audiovisuales.

$\mathrm{Al}$ observar estos términos de forma individual, podemos analizar en profundidad cada uno de sus diferentes contextos, así como los términos con los que se relacionan. Del mismo modo, podemos establecer una serie de conclusiones dentro de nuestra área, generándose nuevos interrogantes, los cuales a su vez constituyen hipótesis de estudio, que pueden dar lugar a nuevas líneas de investigación.

Por otro lado, con la nueva fase de nuestra investigación, conseguiremos detectar también cuáles son los elementos más importantes y en los que incide más cada profesor en su programación. A ello, hay que sumar, que el cuestionario nos revelará interesante información y datos sobre el profesorado en función de los bloques que determinemos.

Hasta el momento, podemos afirmar que la ejecución del análisis realizado por act.musica esquematiza y visualiza perfectamente la representatividad de los elementos constituyentes del currículum musical de la LOE, y próximamente de la programación individual del profesorado de música.

\footnotetext{
${ }^{2}$ No se trata de una aplicación de escritorio.
} 
Las posibilidades que a partir de ahora nos ofrece act.musica son muchas. Así pues, consideramos que mediante una validación cruzada (Russel y Norvig, 2004), se podrían comparar ambos documentos constatándose las diferencias entre la programación y el diseño curricular base. Del mismo modo, no nos cabe duda de que esta herramienta podría encontrar cabida en el marco de otras disciplinas o etapas educativas.

\section{4.- REFERENCIAS BIBLIOGRÁFICAS}

Aguaded, J.I., y Tirado, R. (2008). Los centros tic y sus repercusiones en primaria y secundaria en Andalucía. Educar, 41, 61-90.

Del Rincón, D., Arnal, J., Latorre, A., y Sanz, A (1995). Técnicas de Investigación en Ciencias Sociales. Madrid: Dykinson.

Hopkins, D. (1989). Investigación en el aula. Barcelona: PPU.

Krippendorff, K. (1990). Metodología de análisis de contenido, Barcelona: Paidós.

Ley Orgánica 2/2006, de 3 de mayo, de Educación (BOE nº 106 de 4 de mayo de 2006).

Martín Moreno, M.C. (2002). Las propuestas curriculares para el área de educación musical en la reforma de la LOGSE: antecedentes y estudio comparativo en educación primaria, Tesis Doctoral, Granada: Universidad de Granada.

Orden de 10 de agosto de 2007 por la que se desarrolla el currículo correspondiente a la educación secundaria en Andalucía (BOJA núm. 171 de 30 de agosto de 2007).

Real Decreto 1513/2006, de 7 de septiembre, por el que se establecen las enseñanzas mínimas de la Educación Primaria (BOE num. 293 de 8 de diciembre de 2006).

Real Decreto 1631/2006, de 29 de diciembre por el que se establecen las enseñanzas mínimas correspondientes a la Educación Secundaria (BOE núm. 5 de 5 de enero de 2007).

Rodríguez, S. (2006). El cuestionario. En S. Rodríguez, M.A. Gallardo, M.C. Olmos, y F. Ruiz, Investigación educativa: metodología de encuesta. Granada: GEU.

Ruiz, J.I. (2003) Metodología de la investigación cualitativa, Bilbao: Universidad de Deusto.

Russell, S., y Norvig, P. (2004). Inteligencia Artificial. Un enfoque moderno. Madrid: Pearson.

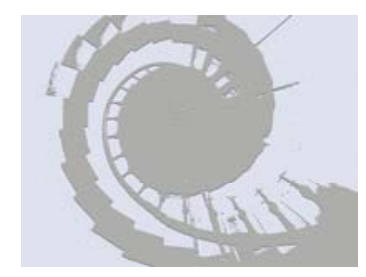

